\title{
Emotionales Leid bei Patienten weit verbreitet
}

\section{Britische Forscher haben Postkarten beginnend den Worten „Liebe Psoriasis ...." an Patienten verteilt, um zu untersuchen, welchen Einfluss die Erkrankung auf die Patienten hat. Diese leiden oft ihr Leben lang unter der Erkrankung und viele fühlen sich von ihren Ärzten nicht ernstgenommen. Die Ärzte hingegen klagen häufig über mangelnde Therapietreue. $\mathrm{Br}$ J Dermatol 2014; 171: 825-831}

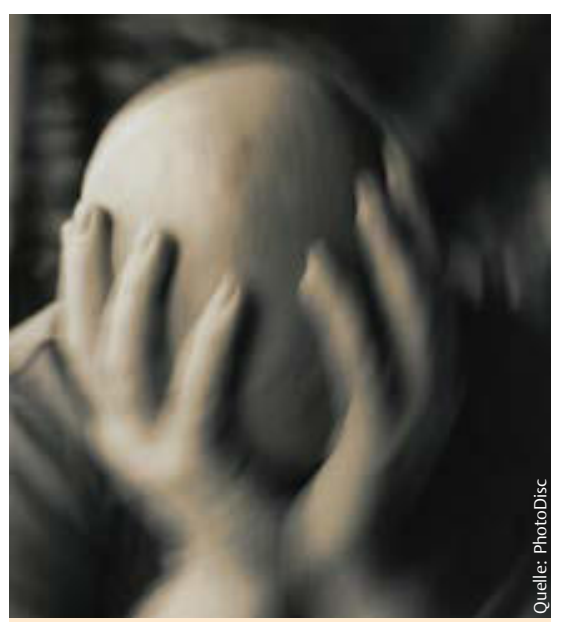

Viele Psoriasis-Patienten leiden unter erheblichen psychischen Belastungen.

Um Patienten mit Psoriasis besser unterstützen zu können, ist es hilfreich, die inneren Bilder zu erfassen, die Betroffene von ihrer Krankheit haben. Sie leiden nicht nur an ihrer wunden, juckenden und schuppigen Haut - viele fühlen sich dauerhaft angespannt, können Gefühle nur schwer in Worte fassen und weisen ein erhöhtes Suizidrisiko auf. C. Bundy und Kollegen der Universität Manchester, Großbritannien, haben Psoriasis-Patienten die Möglichkeit gegeben, ihre Erkrankung auf einer Postkarte mit dem Eingangssatz „Liebe Psoriasis ...“ darzustellen. Sie verschickten rund 2100 Postkarten an die Mitglieder der britischen Psoriasis Association. Außerdem legten sie Postkarten im Wartezimmer der dermatologischen Studien-Ambulanz aus. Alter und Geschlecht der Patienten spielten keine Rolle. Die Patienten konnten ihre Karten anonym abgeben. Entscheidend waren allein die Gedanken, Gefühle und Bilder zur Erkrankung.

\section{Ein ständiger Kampf \\ $\nabla$}

Es kristallisierten sich 7 Themen heraus, die sich wiederholten: Psoriasis beeinflusst die Identität und Beziehungen, sie ist ein Kampfplatz, sie zerstört das Gefühl von Kontrolle, sie hat emotionale Konsequenzen, sie führt zur erhöhten Wachsamkeit, zu sarkastischen Coping-Strategien sowie zu psychischem Leid durch die Behandlung.

Während manche Patienten die Psoriasis als ihren Freund betrachten, sehen andere in ihr nur den Feind. Besonders die lange Zeitspanne der Erkrankung beeinträchtigt die Patienten. Auf einer Postkarte steht: „Du bist wie ein Ehemann! 40 Jahre sind wir zusammen und ich verstehe Dich nicht.“ Auf einer anderen Karte ist zu lesen: „Du bist mein Peiniger seit ich 11 Jahre alt bin“, oder: „Der Feind in mir.“

Viele Betroffene werden durch die Erkrankung in eine Außenseiterposition gedrängt. Andere Menschen ekeln sich vor ihren Hauterscheinungen oder halten sie gar für ansteckend. Auf den Postkarten steht: „Wer würde mich heiraten oder mit mir schlafen wollen?“, oder: „Ich wurde direkt isoliert, weil die Hebammen dachten, ich wäre infektiös - wie beschämend!“, oder: „Mein Hausarzt interessiert sich nicht und das trägt zu meinem geringen Selbstwertgefühl bei." Auch fühlen sich die Patienten extrem hilflos und in einem ständigen Kampf: „Egal, was ich mache: Wenn Du entscheidest, dass Du aufflackern willst, wirst Du es tun“, schreibt ein Betroffener, während jemand anderes schreibt: „Du bist immer da und ich werde immer an Dich erinnert.“
Nicht zuletzt ist es die Behandlung, die die Patienten bedrückt. Eine Postkarte zeigt: „Die Psoriasis hat meine Kindheit und Teenager-Jahre geformt. 4-6 Stunden musste ich täglich für meine Behandlung aufwenden (eine übel riechende war es zudem). Da gab es die langen, juckenden und schmerzenden Nächte und die 12-bis 18-wöchigen Aufenthalte im Krankenhaus ..." Die Postkarten lassen nur erahnen, welcher Belastung die Patienten ausgesetzt sind. Die Autoren halten weitere Forschungen auf diesem Gebiet für sinnvoll - auch, um vulnerable Patientengruppen zu identifizieren.

\section{Fazit}

Psoriasis-Patienten stellten auf Postkarten eindrucksvoll dar, welchen immensen Einfluss die Erkrankung auf ihr Leben hat: Sie beeinflusst sowohl die eigene Identität als auch die Beziehungen. Die Patienten erleben die Psoriasis wie einen ständigen Kampf, über den sie keine Kontrolle haben. Ihr Selbstwertgefühl ist meist stark beeinträchtigt. Die Studie zeigt, wie groß die Not der Patienten ist, sodass eine psychologische Begleitung als Teil der Psoriasis-Behandlung für viele Betroffene sinnvoll sein könnte, so die Autoren

\section{Dr. Dunja Voos, Pulheim}

\section{Ausschreibung}

\section{Fleur Hiege- Gedächtnispreis}

Die Hiege-Stiftung schreibt den mit $10000 €$ dotierten Fleur Hiege-Gedächtnispreis 2015 aus. Ausgezeichnet werden herausragende Forschungsleistungen bei der Bekämpfung des Hautkrebses, insbesondere des Melanoms. Die Ausschreibungsfrist endet am 30. Juni 2015. Bewerber können sich mit einer Forschungsarbeit, die innerhalb der beiden letzten Jahre publiziert bzw. zur Publikation angenommen wurde und ganz oder weitgehend an einer deutschen Klinik/einem deutschen Institut angefertigt wurde, bei der HiegeStiftung gegen Hautkrebs, Parkallee 43 in 20144 Hamburg bewerben. Die Bewerber sollten zum Zeitpunkt der Einreichung das 40. Lebensjahr nicht überschritten haben.

Nach einer Mitteilung

der Hiege-Stiftung, Hamburg 